\title{
OBESIDAD PREGESTACIONAL Y RIESGO DE INTOLERANCIA A LA GLUCOSA EN EL EMBARAZO Y DIABETES GESTACIONAL
}

\author{
Pamela Nava D. 1,a , Adriana Garduño A., MSc 1,4,a, Silvia Pestaña M. ${ }^{2}$, Mauricio San- \\ tamaría F. ${ }^{2}$, Gilberto Vázquez DA., PhD ${ }^{3,4}$, Roberto Camacho B. ${ }^{1}$, Javier Herrera V., \\ MSc. $^{1}$ \\ ${ }^{1}$ Unidad de Investigación, ${ }^{2}$ División de Medicina Materno Fetal, ${ }^{3}$ Departamento de Robótica, Hospital Materno Perina- \\ tal "Mónica Pretelini”, ISEM. ${ }^{4}$ Centro de Investigación en Ciencias Médicas, CICMED. Toluca, México.
}

a Nutricionista

\section{RESUMEN}

Antecedentes: La obesidad en mujeres en edad reproductiva es cada vez más frecuente y complica el embarazo aumentando el riesgo de enfermedades como la diabetes mellitus gestacional (DMG). Objetivo: Determinar el riesgo de presentar DMG e intolerancia a la glucosa (ITG) de acuerdo con el índice de masa corporal pregestacional (IMCPG). Método: Muestra de 489 pacientes, se clasificaron por IMCPG y se les realizó una curva de tolerancia oral a la glucosa (CTOG) de $100 \mathrm{~g}$ de 120 minutos. Se definió DMG con dos valores alterados en la CTOG e ITG con un solo valor alterado. Se aplicó prueba de chi cuadrada para determinar diferencia entre grupos y se obtuvo un Odds Ratio (OR), con intervalo de confianza del $95 \%$. Resultados: 9 pacientes con bajo peso, 194 con peso normal, 158 con sobrepeso y 128 con obesidad, edad gestacional promedio $31,3 \pm 5,6$ semanas. El $13 \%$ de las pacientes presentaron DMG, el 10,6\% ITG. Las mujeres con sobrepeso presentaron un OR de 3,81 para desarrollar ITG (IC95\% 1,62-8,95) y un OR de 3,7 para DMG (IC95\% 1,65-8,38), mientas que las pacientes con obesidad pregestacional presentaron un OR de 6,6 para desarrollar ITG (IC95\% 2,83-15,66) y un OR de 8,8 para DMG (IC95\% 4,05-19,51), comparadas con mujeres con peso pregestacional normal. Conclusión: La población mexicana tiene mayor riesgo de DMG que otras poblaciones y mayor prevalencia de sobrepeso y obesidad, por lo que se debe realizar un diagnóstico y tratamiento oportuno de DMG, previniendo complicaciones y diabetes mellitus tipo 2.

\section{PALABRAS CLAVE: Embarazo, diabetes gestacional, obesidad, sobrepeso}

\section{SUMMARY}

Background: Obesity in women in reproductive age is becoming more frequent and it self complicates pregnancy increasing the risk of diseases such as gestational diabetes mellitus (GDM). Aims: To determine the risk of gestational diabetes mellitus (GDM) and impaired glucose tolerance (IGT) according to the pregestational body mass index (PGBMI). Methods: Sample of 489 patients, they were classified by PGBMI and underwent an oral glucose tolerance test (OGTT) with $100 \mathrm{~g}$ of glucose during 120 minutes. GDM was defined with two altered values in the OGTT and IGT with a single altered value. A chi-square test was applied to determine difference between groups, and we obtained an Odds Ratio (OR), with a confidence level of 95\%. Results: 9 patients were low weight, 194 normal weight, 158 overweight and 128 obese, average gestational age $31.3 \pm 5.6$ weeks. $13 \%$ of the patients had GDM, $10.6 \%$ had IGT. Overweight women had an OR of 3.81 of developing IGT (95\% Cl 1.62-8.95) and an OR of 3.7 of developing GDM (95\% Cl 1.65-8.38), while women with prepregnancy obesity showed an OR of 6.6 of developing ITG (95\% Cl 2.83-15.66) and 
an OR of 8.8 of developing GDM $(95 \% \mathrm{Cl} 4.05-19.51)$, all compared to women with prepregnancy normal weight. Conclusions: Mexican population has a higher risk of GDM than other populations and also a higher prevalence of overweight and obesity, so that every pregnant woman should be screening for GDM, has a diagnosis and receive treatment, preventing complications and Type 2 Diabetes.

\section{KEY WORDS: Pregnancy, gestational diabetes, obesity, overweight}

\section{INTRODUCCIÓN}

La obesidad es una enfermedad caracterizada por exceso de tejido adiposo, definida por un índice de masa corporal (IMC) mayor o igual a 30. En México, el $34,5 \%$ de las mujeres mayores de 20 años son obesas y el 37,4\% tienen sobrepeso, es decir, más del $70 \%$ de las mujeres mexicanas tienen sobrepeso u obesidad, de las cuales el 7,2\% padecen diabetes mellitus tipo 2 (DM2) $(1,2)$.

La obesidad se ha asociado con un elevado riesgo de padecer diabetes mellitus gestacional (DMG) $(3,4)$. El índice de masa corporal pregestacional (IMCPG) tiene mayor asociación que la ganancia de peso gestacional elevada con padecer DMG e intolerancia a la glucosa en el embarazo (ITG) $(3,5,6)$. El embarazo es considerado un estado diabetogénico, y comenzarlo con sobrepeso $u$ obesidad origina un aumento de la resistencia a la insulina, lo que ocasiona agotamiento de la capacidad de las células $B$ de secretar la cantidad de insulina requerida por el embarazo, aumentando el riesgo de desarrollar DMG $(3,4)$.

La DMG complica del 8 al $12 \%$ de los embarazos en México (7). Tanto la DMG como la presencia de hiperglucemia materna se relacionan con complicaciones perinatales y con un alto riesgo de desarrollar obesidad y DM2, posteriormente, tanto en la madre como en el niño (4,5,8-10).

Estudios estiman que el riesgo de desarrollar DMG aumenta considerablemente entre mujeres embarazadas conforme aumenta el IMC $(11,12)$. En nuestra población, considerada de alto riesgo para desarrollar DM (13), no está bien definida la asociación de DMG con el estado de nutrición previo al embarazo, por lo que el objetivo de nuestro estudio es determinar el riesgo de presentar DMG e ITG de acuerdo con el IMCPG.

\section{PACIENTES Y MÉTODOS}

Estudio de casos y controles, prospectivo, transversal. Se estudió una muestra de 489 mujeres embarazadas del Hospital Materno Perinatal "Mónica Pretelini", Toluca, México, de enero de 2009 a marzo de 2010.
Se incluyeron pacientes de cualquier edad gestacional, de 13 a 44 años de edad. Se excluyeron pacientes con diagnóstico previo de DM2; se eliminaron aquellas que no completaron la prueba o que no toleraron la solución glucosada. Todas las pacientes contaron con servicio médico gratuito.

A cada paciente se le realizó un registro de datos que incluían edad, peso pregestacional, peso y talla (se pesaron y midieron en una báscula con estadímetro marca SECA $700 \AA$ ), gestas, antecedentes familiares de diabetes, enfermedades agregadas y los resultados de la prueba. En caso de no recordar el peso pregestacional, se buscó el dato en el carnet de consultas prenatales de cada paciente.

Según índice de masa corporal pregestacional (IMCPG) se definió como embarazada de bajo peso a un IMC $<18,5$; normal IMC 18,5 - 24,9; sobrepeso IMC $25-29,9$ y obesidad IMC $\geq 30$.

Se les realizó una curva de tolerancia oral a la glucosa para diagnóstico elaborada y donada por Laboratorios Silanes $\AA$ (100 g de glucosa anhidra en $300 \mathrm{ml}$ de agua), con determinación de 3 valores de glucosa sérica: en ayuno, a los 60 minutos y a los 120 minutos poscarga. Se omitió la toma de la tercera hora, ya que está demostrado que en nuestra población, la CTOG de $100 \mathrm{~g}$ sin éste valor tiene una sensibilidad del $91,2 \%$ (14). Se obtuvo una muestra de sangre venosa de $5 \mathrm{ml}$ para determinaciones bioquímicas, en condiciones de ayuno de 12 horas. La glucosa se determinó por técnicas enzimáticas utilizando el equipo Dimension $®$ Clinical Chemestry System, SIEMENS (Alemania). Se definió DMG con dos valores alterados en la CTOG e ITG con un solo valor alterado en la prueba (15).

Para el análisis estadístico se utilizó el paquete SPSS versión 17. Se aplicó la prueba de chi cuadrada para determinar diferencias entre los grupos. El análisis de riesgo se efectuó mediante el cálculo de Odds Ratio (OR) con un intervalo de confianza (IC) del 95\%.

El estudio fue aprobado por el comité de ética del hospital. No se requirió carta de consentimiento informado, ya que de acuerdo con la Norma Oficial Mexicana NOM 015, se debe realizar escrutinio de DMG en nuestra población (16). 


\section{RESULTADOS}

Las características descriptivas de la población clasificada de acuerdo a su IMCPG, así como las principales patologías agregadas al momento de la prueba se presentan en la Tabla I.

De las 489 pacientes incluidas, el 1,8\% presentaron bajo peso, el $39,6 \%$ peso normal, el $32,3 \%$ sobrepeso y el $26,1 \%$ obesidad, clasificadas con los criterios del Institute of Medicine (IOM) para embarazo (17). La edad promedio por grupo fue: para bajo peso de $19 \pm 4,2$ años, para peso normal $24,1 \pm 6,7$ años, para sobrepeso $28,7 \pm 7,2$ años y para obesidad 29,4 $\pm 6,5$ años. La edad gestacional promedio fue de $31,3 \pm 5,6$ semanas. Del total, el $13 \%$ de las pacientes presentaron DMG, el 10,6\% presentaron ITG. De las 64 pacientes con DMG, el 51,5\% fueron obesas y el $34,3 \%$ presentaron sobrepeso, el resto de las frecuencias y porcentaje de pacientes con DMG e ITG se observan en la Tabla II.

El riesgo (OR, IC95\%) de desarrollar DMG o ITG de acuerdo con el IMCPG se observan en la Tabla III. Hubo un significativo $(p<0,001)$ mayor riesgo de DMG o ITG en las embarazadas con sobrepeso u obesidad pregestacional respecto a las con IMC normal.

\section{DISCUSIÓN}

El impacto de la obesidad durante el embarazo no está del todo descrito en la población mexicana. De la población incluida en nuestro estudio, el $32,3 \%$ tuvo sobrepeso pregestacional y el $26,1 \%$ obesidad, es decir, más del $50 \%$ de las mujeres embarazadas que se sometieron a la CTOG tienen sobrepeso u obesidad pregestacional. La edad promedio en este grupo de pacientes es mayor que en las que presentan bajo peso y peso normal pregestacional, así como también se observó un aumento en la frecuencia de multiparidad en el grupo de sobrepeso y obesidad, lo cual concuerda con la descripción de factores de riesgo para presentar DMG (13). De acuerdo con nuestros resultados, las mujeres con sobrepeso pregestacional tienen 3,8 veces más riesgo de ITG y 3,7 veces más riesgo de DMG, mientas que las que padecen obesidad pregestacional presentan 6,6 veces más riesgo de ITG y 8,8 veces más riesgo de DMG, comparadas con mujeres que inician el embarazo con peso normal.

Los resultados obtenidos en el estudio concuerdan con estudios previos que asocian a la obesidad como un factor de riesgo de DMG $(13,18,19)$. Kim y cols (12), reportan que los porcentajes de DMG atribuibles al sobrepeso, obesidad y obesidad extrema pregestacional son del $15,4 \%, 9,7 \%$ y $21,1 \%$ respectivamente. Nuestros resultados respecto al riesgo de desarrollar DMG son similares a los reportados por Chu y cols (11), sin embargo, en su metanálisis no incluyen estudios realizados exclusivamente en población latina, y clasifican a la obesidad en dos grupos (obesidad y obesidad extrema); nuestro OR de DMG para obesidad fue mayor que el obtenido por ellos (IMCPG 30-35, OR 3,56), y es similar al que obtuvieron para obesidad extrema (IMCPG >35, OR 8,56), lo cual puede deberse a que incluyen población caucásica considerada de bajo riesgo.

Tabla I

CARACTERÍSTICAS DE LA POBLACIÓN DE ACUERDO A SU IMCPG

\begin{tabular}{|c|c|c|c|c|}
\hline & $\begin{array}{c}\text { Bajo peso } \\
n=9\end{array}$ & $\begin{array}{l}\text { Normal } \\
n=194\end{array}$ & $\begin{array}{c}\text { Sobrepeso } \\
n=158\end{array}$ & $\begin{array}{c}\text { Obesidad } \\
n=128\end{array}$ \\
\hline Primigestas $\mathrm{n}(\%)$ & $7(77,7)$ & $95(48,9)$ & $43(27,2)$ & $28(21,8)$ \\
\hline Multigestas $\mathrm{n}(\%)$ & $2(22,2)$ & $99(51,3)$ & $115(72,7)$ & $100(78,1)$ \\
\hline Antecedentes DM2 n (\%) & $3(33,3)$ & $107(55,1)$ & $101(63,9)$ & $88(68,7)$ \\
\hline \multicolumn{5}{|l|}{ Enfermedades durante el embarazo n (\%) } \\
\hline$\bullet$ Enfermedad cardiaca & $1(11,1)$ & $2(1,0)$ & $3(1,8)$ & 0 \\
\hline$\bullet$ HASC & 0 & $5(2,5)$ & $7(4,4)$ & $5(3,9)$ \\
\hline - HA inducida por el embarazo & 0 & $3(1,5)$ & $6(3,7)$ & $3(2,3)$ \\
\hline - Hipotiroidismo & 0 & $3(1,5)$ & $2(1,2)$ & 0 \\
\hline - Hipertiroidismo & $1(11,1)$ & $1(0,5)$ & $2(1,2)$ & 0 \\
\hline
\end{tabular}

IMCPG: índice masa corporal pregestacional. HASC: hipertensión arterial crónica. HA: hipertensión arterial 
Tabla II

FRECUENCIA DE DMG E ITG DE ACUERDO CON EL IMCPG.

\begin{tabular}{|c|c|c|c|c|c|c|c|c|c|c|}
\hline \multirow[b]{2}{*}{ CTOG } & \multicolumn{2}{|c|}{$\begin{array}{c}\text { Bajo Peso } \\
n=9 \\
(1,8 \%)\end{array}$} & \multicolumn{2}{|c|}{$\begin{array}{c}\text { Normal } \\
n=194 \\
(39,6 \%)\end{array}$} & \multicolumn{2}{|c|}{$\begin{array}{c}\text { Sobrepeso } \\
n=158 \\
(32,3 \%)\end{array}$} & \multicolumn{2}{|c|}{$\begin{array}{c}\text { Obesidad } \\
n=128 \\
(26,1 \%)\end{array}$} & \multicolumn{2}{|c|}{$\begin{array}{c}\text { Total } \\
n=489 \\
(100 \%)\end{array}$} \\
\hline & $\mathrm{n}$ & $\%$ & $\mathrm{n}$ & $\%$ & $\mathrm{n}$ & $\%$ & $n$ & $\%$ & $\mathrm{n}$ & $\%$ \\
\hline 1 valor alterado* & 1 & 11,1 & 8 & 4,1 & 20 & 12,6 & 22 & 17,1 & 51 & 10,4 \\
\hline$(+) \mathrm{DMG}^{\star \star}$ & 0 & 0 & 9 & 4,6 & 22 & 13,9 & 33 & 25,7 & 64 & 13,0 \\
\hline$(-)^{\star \star \star}$ & 8 & 88,8 & 177 & 91,2 & 116 & 73,4 & 73 & 57,1 & 374 & 76,4 \\
\hline
\end{tabular}

DMG: diabetes mellitus gestacional. CTOG: curva de tolerancia oral a la glucosa. ITG: intolerancia a la glucosa. IMCPG: índice de masa corporal pregestacional. *Intolerancia a la glucosa en el embarazo. ${ }^{* \star}$ Diabetes mellitus gestacional. ${ }^{* \star \star}$ CTOG con todos los valores en rango normal.

Tabla III

RIESGO DE DMG EN NUESTRA POBLACIÓN DE ACUERDO CON SU IMCPG

\begin{tabular}{|c|c|c|c|c|c|c|}
\hline & \multicolumn{2}{|c|}{ Normal } & \multicolumn{2}{|c|}{ Sobrepeso } & \multicolumn{2}{|c|}{ Obesidad } \\
\hline & $\mathrm{n}=202$ & OR (IC95\%) & $n=104$ & OR (IC95\%) & $n=152$ & OR (IC95\%) \\
\hline Intolerancia a la glucosa & 8 & --- & 20 & $3,8(1,62-8,95)^{a}$ & 22 & $6,6(2,83-15,66)^{a}$ \\
\hline Diabetes mellitus gestacional & 9 & --- & 22 & $3,7(1,65-8,38)^{a}$ & 33 & $8,8(4,05-19,51)^{a}$ \\
\hline
\end{tabular}

a valor $\mathrm{p}<0,001$ comparado con el grupo normal.

El riesgo encontrado en nuestra población es similar aunque menor al reportado por El-Gilany y Hammad (20), en mujeres con obesidad pregestacional en Arabia Saudita (utilizando la misma clasificación de IMC que nosotros), quienes describen un OR de 9,3 en mujeres obesas comparado con mujeres con peso normal, (IC95\%: 2,717-31,84); para mujeres con sobrepeso el OR fue de 4,5 (IC95\%: 1,186-17,29). Yeung y cols (21), refieren que la ganancia de peso durante la edad adulta, así como la obesidad pregestacional, están más fuertemente asociadas a padecer DMG que la obesidad en otras etapas de la vida y otros factores de riesgo conocidos, resaltando un riesgo aumentado cuando existe obesidad central en la adultez. La obesidad en áreas localizadas como la abdominal no fue medida en nuestro estudio.

Una de las limitaciones de nuestro estudio fue no contar con la ganancia de peso gestacional en la población estudiada. De acuerdo con la Asociación Americana de Diabetes, la ganancia de peso durante la gestación es un fuerte factor de riesgo para desarrollar DMG, incluso en pacientes con obesidad pregestacional (22). Sin embargo, Zonana-Nacach y cols (5), encontraron que el riesgo de DMG en mujeres mexicanas en Tijuana, BC, no tiene asociación con la ganancia de peso (OR: $1,0)$, pero la frecuencia aumenta claramente con- forme aumenta el IMCPG. En nuestro estudio no evaluamos el riesgo de presentar DMG en mujeres embarazadas con obesidad pregestacional con una adecuada ganancia de peso comparadas con aquellas que además tuvieron exceso de ganancia de peso, lo cual podría considerarse para estudios posteriores

Nuestros resultados apoyan que la prevención de DMG depende en gran medida de la prevención de obesidad en mujeres jóvenes. Del 15 al $60 \%$ de las mujeres con DMG desarrollarán DM2 en un lapso de 5 a 15 años después de la resolución del embarazo, e incluso aquellas que solo presentaron ITG, probablemente también evolucionen a DM2 y síndrome metabólico (8-10). Prevenir DM2 en mujeres obesas con DMG e ITG depende de las intervenciones efectivas en materia de nutrición y actividad física que logren una pérdida de peso y del diagnóstico y tratamiento oportuno de la población en riesgo $(5,11,13)$.

\section{CONCLUSIONES}

La embarazada con sobrepeso $u$ obesidad tiene alto riesgo de desarrollar DMG e ITG, por lo que es imprescindible realizar una CTOG, para realizar un diagnóstico y tratamiento oportuno, previniendo complicaciones y reduciendo el riesgo de desarrollar 
DM2 mediante intervenciones con un equipo multidisciplinario. La población mexicana tiene un mayor riesgo de DMG al reportado para otras poblaciones, y deben tomarse medidas preventivas efectivas tanto para obesidad como para DM2.

\section{BIBLIOGRAFÍA}

1. Norma oficial mexicana, NOM-008-SSA3-2010. Para el tratamiento integral del sobrepeso y la obesidad. Disponible en: http://www.dof.gob.mx/nota_detalle.ph $\mathrm{p}$ ?codigo $=5154226 \&$ fecha $=04 / 08 / 2010$ [Consultada: 30 de Septiembre 2010].

2. Encuesta Nacional de Salud y Nutrición 2006, Instituto Nacional de Salud Pública. Disponible en: http://www. insp.mx/encuesta-nacional-salud-y-nutricion-2006. html [Consultada: 30 de Septiembre 2010].

3. Hedderson MM, Williams MA, Holt VL, Weiss NS, Ferrara A. Body mass index and weight gain prior to pregnancy and risk of gestational diabetes mellitus. Am J Obstet Gynecol 2008;198:409.e1-409.e7.

4. Herring SJ, Oken E, Rifas-Shiman SL, Rich- Edwards JW, Stuebe AM, Kleinman KP, et al. Weight gain in pregnancy and risk of maternal hyperglycemia. Am J Obstet Gynecol 2009;201:e1-7.

5. Zonana-Nacach A, Baldenebro-Preciado R, RuizDorado MA. Efecto de la ganancia de peso gestacional en la madre y el neonato. Salud Publica Mex 2010;52:220-5.

6. De la Calle FMM, Armijo LO, Martín BE, Sancha NM, Magdaleno DF, Omeñaca FT, et al. Sobepeso y obesidad pregestacional como factor de riesgo de cesárea y complicaciones perinatales. Rev Chil Obstet Ginecol 2009;74:233-8.

7. Muñoz SR, Reyes PN, Barranco JA, Sánchez FI. Diabetes y Embarazo. Boletín de Práctica Médica Efectiva, INSP - SSA, 2007.

8. Baptise-Roberts K, Barone B, Gary T, Golden SH, Wilson LM, Bass EB, et al. Risk Factors for type 2 diabetes among women with gestational diabetes: A systematic review. Am J Med 2009;22:207-14.

9. Corrado F, D'Anna R, Cannata ML, Cannizaro D, Caputo F, Raffone E, et al. Positive association between a single abnormal glucose tolerance test value in pregnancy and subsequent abnormal glucose tolerance. Am J Obstet Gynecol 2007;196:339.e1-339.e5.

10. Retnakaran R. Glucose intolerance in pregnancy and postpartum risk of metabolic syndrome in young women. J Clin Endocrinol Metab 2010;95:670-7.

11. Chu SY, Callaghan WM, Kim SY, Schimd CH, Lau J, England LJ, Dietz P, Maternal obesity and risk of gestational diabetes mellitus. Diabetes Care 2007;30:2070-6.

12. Kim SY, England L, Wilson HG, Bish C, Satten GA, Dietz $P$, Percentage of gestational diabetes mellitus attributable to overweight and obesity. Am J Public Health 2010;100:1047-52.

13. Standars of Medical Care in Diabetes 2010, Position statement of the American Diabetes Association. Diabetes Care 2010;33(suppl 1).

14. Ortega GC, Ballesteros A, Casanueva E, Fonseca T, Casique E, Parra A, Searching for alternative methods of diagnosing gestational diabetes mellitus in a Mexican urban population. Med Sci Monit 2008;14:CR598603.

15. American Diabetes Association (ADA). Gestational diabetes mellitus. Position statement. Diabetes Care 2004;27:S88-S90.

16. NORMA OFICIAL MEXICANA, NOM-015-SSA2-1994. Para la prevención, tratamiento y control de la diabetes mellitus en la atención primaria. Disponible en: http:// www.salud.gob.mx/unidades/cdi/nom/015ssa24.html [Consultada: 04 de Septiembre 2010].

17. Institute of Medicine (IOM). Rasmussen KM, Yaktine AL (Editors). Weight Gaing During Pregnancy IOM. Reexamining the Guidelines, National Academies Press [serial on the Internet], 2009.

18. Athukorala C, Rumbold RA, Willson K, Crowther CA. The risk of adverse pregnancy outcomes in women who are overweight or obese. BMC Pregnancy Childbirth 2010;10:1-8.

19. Guelinckx I,Devlinger R, Beckers K, Vansant G. Maternal Obesity: pregnancy complications, gestational weight gain and nutrition. Obesity Reviews 2008;9:140-50.

20. El-Gilany AH, Hammad S. Body Mass Index and Obstetric Outcomes in Saudi Arabia: a prospective cohort study. Ann Saudi Med 2010;30:376-80.

21. Yeung EH, Hu FB, Solomon CG, Chen L, Louis GM, Schisterman $\mathrm{E}$, et al. Life course weight characteristics and the risk of gestational diabetes. Diabetologia 2010;53:668-78.

22. Bloomgarden ZT. Gestational diabetes mellitus and obesity. Diabetes Care 2010;33:e60-e65. 